\title{
Avaliação dos testes de compressão da resina acrílica termopolimerizável quando incluído o poli (cloreto de dialildimetilamônio) em sua massa
}

\author{
Evaluation of compression tests of thermopolymerizable acrylic resin when poly \\ (diallyldimethylammonium chloride) is included in its mass \\ Evaluación de las pruebas de comprensión de la resina acrílica termopolimerizable cuando incluido \\ el (cloreto de dialildimetilamonio) en su massa
}

Recebido: 30/04/2021 | Revisado: 06/05/2021 | Aceito: 07/05/2021 | Publicado: 21/05/2021

Lucas Laion da Silva Oliveira

ORCID: https://orcid.org/0000-0001-8348-3885

Universidade Anhanguera, Brasil

E-mail: lucaslayon03@hotmail.com

Daniela Coluce Herrerias Gualberto

ORCID: https://orcid.org/0000-0002-8927-6585

Universidade Anhanguera, Brasil

E-mail: dhgualberto@gmail.com

Neide Pena Coto

ORCID: https://orcid.org/0000-0002-3235-0684 Universidade de São Paulo, Brasil

E-mail: npcoto@usp.br

Carina Domaneschi

ORCID: https://orcid.org/0000-0001-8615-3283

Universidade de São Paulo, Brasil

E-mail: domaneschi@usp.br

Rafael Traldi Moura

ORCID: https://orcid.org/0000-0002-4977-239X

Universidade de São Paulo, Brasil

E-mail: moura.gmsie.usp@gmail.com

Syrio Simão Netto

ORCID: https://orcid.org/0000-0003-2795-4755

Universidade Anhanguera, Brasil

E-mail: ssimaonetto@uol.com.br

Rennan Luiz Oliveira dos Santos

ORCID: https://orcid.org/0000-0001-9093-3805

Universidade Anhanguera, Brasil

E-mail: rennan_475@hotmail.com

\begin{abstract}
Resumo
Introdução: As resinas acrílicas termopolimerizáveis são utilizadas na odontologia de maneira bastante difundida por possuírem diversas propriedades satisfatórias. Esses materiais tendem a sofrer modificações em sua estrutura durante o seu uso podendo causar inflamações e infecções. As mais diversas pesquisas buscaram desenvolver um meio de diminuir essa possibilidade e Dos Santos em 2020 evidenciou que esse material acrescido do biocida poli(cloreto de dialildimetilamônio) tem um potencial antibacteriano e antifúngico, indicando esse acréscimo apenas para próteses que não sofram algum tipo de força durante seu uso. Objetivo: evidenciar o comportamento da resina acrílica termopolimerizada acrescida do poli(cloreto de dialildimetilamônio) frente a testes de compressão buscando avaliar o seu comportamento frente a essa situação. Métodos: Os testes de compressão foram realizados utilizando a maquina de ensaio universal Instron3369 de coluna dupla frente a dois grupos; sendo G1 o material sem a adição do biocida e G2 o material com a adição do biocida. Resultados: Os resultados demonstraram que G2 sofre mais deformação exigindo uma força de compressão menor que G1. Conclusão: Fica evidente que há prejuízos nas propriedades mecânicas da resina acrílica termopolimerizada acrescida do poli(cloreto de dialildimetilamônio), mas que é necessário mais testes para uma futura utilização ou não desse material em diferentes locais.
\end{abstract}

Palavras-chave: Resinas acrílicas; Biocida; Materiais dentários.

\section{Abstract}

Introduction: Thermopolymerizable acrylic resins are widely used in dentistry because they have several satisfactory properties. These materials tend to undergo changes in their structure during use, which can cause inflammation and infections. The most diverse researches sought to develop a way to reduce this possibility and Dos Santos in 2020 
evidenced that this material added with the biocide poly (diallyl dimethylammonium chloride) has an antibacterial and antifungal potential, indicating this increase only for prostheses that do not suffer any type of force during its use. Objective: to show the behavior of the thermopolymerized acrylic resin plus poly (diallyl dimethylammonium chloride) against compression tests seeking to evaluate its behavior in this situation. Methods: The compression tests were carried out using the double-column Instron3369 universal testing machine in front of two groups; G1 being the material without the addition of the biocide and G2 the material with the addition of the biocide. Results: The results showed that G2 undergoes more deformation requiring less compression force than G1. Conclusion: It is evident that there are losses in the mechanical properties of the thermopolymerized acrylic resin plus poly (diallyl dimethylammonium chloride), but that further tests are necessary for the future use or not of this material in different locations.

Keywords: Acrylic resins; Biocide; Dental materials.

\section{Resumen}

Introducción: Las resinas acrílicas termopolimerizables se utilizan en odontología de forma muy extendida porque tienen varias propiedades satisfactorias. Estos materiales tienden a sufrir cambios en su estructura durante su uso, lo que puede provocar inflamación e infecciones. Las más diversas investigaciones buscaron desarrollar una forma de reducir esta posibilidad y Dos Santos en 2020 mostró que este material agregado con el biocida poli (cloruro de dialildimetilamonio) tiene un potencial antibacteriano y antifúngico, indicando este aumento solo para prótesis que no sufren ningún tipo. de fuerza durante su uso. Objetivo: mostrar el comportamiento de la resina acrílica termopolimerizada más poli (cloruro de dialildimetilamonio) frente a ensayos de compresión buscando evaluar su comportamiento en esta situación. Métodos: Las pruebas de compresión se realizaron utilizando la máquina de prueba universal de doble columna Instron3369 frente a dos grupos; G1 es el material sin la adición del biocida y G2 el material con la adición del biocida. Resultados: Los resultados mostraron que G2 sufre más deformaciones requiriendo menos fuerza de compresión que G1. Conclusión: Es evidente que existen pérdidas en las propiedades mecánicas de la resina acrílica termopolimerizada más poli (cloruro de dialildimetilamonio), pero que son necesarias más pruebas para el uso futuro o no de este material en diferentes ubicaciones.

Palabras clave: Resinas acrílicas; Biocida; Materiales dentales.

\section{Introdução}

As resinas acrílicas termopolimerizáveis são comumente indicadas para a confecção de próteses bucomaxilofaciais, intra e extra orais, por possuir propriedades mecânicas, físicas e químicas que contemplam a maioria das necessidades impostas pelo uso de tais próteses (Santos et al., 2016; Coto et al., 2017). Entretanto, quando expostas a ação do tempo, fluidos, alimentos e produtos de higiene sofrem desgaste e modificação estrutural, favorecendo o crescimento de microrganismos podendo causar inflamação e infecção no local (Rezende, 1997; Turano JC et al., 2010; Dos Santos, 2020).

O uso de hipoclorito de sódio e clorexidina, por exemplo, não são indicados, pois podem acarretar a possibilidade de clareamento da resina acrílica ou corrosão de componentes. A busca pela solução deste problema visando inibir proliferação de microrganismos em resinas acrílicas utilizadas na confecção de prótese bucomaxilofaciais tem sido realizadas. (Da Silva, 2009; Silva, 2012; Peracini, 2012; Amal et al., 2016; Rocha et al., 2017).

Dos Santos em suas pesquisas (Dos Santos, 2016; Dos Santos et al., 2019; Dos Santos 2020) constatou uma considerável eficiência antibacteriana e antifúngica do poli(cloreto dialildimetilamônio) incluído na massa das resinas acrílicas, além da sua biocompatibilidade, indicando esse material para próteses que não realizam esforços mastigatórios.

Porém sabe-se que as resinas acrílicas termopolimerizadas são utilizadas na confecção de próteses intraorais e que essas próteses são submetidas a longos períodos de esforço mastigatório e, portanto, podem sofrer fraturas (Eisenburger M et al., 2008; Straioto FG et al., 2010). Visto que atualmente, as resinas acrílicas vêm sendo aprimoradas em suas características alcançando melhores propriedades e, consequentemente, uma melhor aplicabilidade durante seu uso, este trabalho visou a uma possível melhora de resinas termopolimerizaveis de uso em próteses intraorais, avaliando seu desempenho mecânico quando acrescidas do biocida poli(cloreto dialildimetilamônio). 


\section{Metodologia}

Trata-se de uma pesquisa laboratorial e quantitativa. Seguindo a metodologia de Dos Santos em 2020, foram confeccionados 12 corpos de provas, preparados no Laboratório de Pesquisa do Departamento de Estomatologia da Faculdade de Odontologia da Universidade de São Paulo, com a resina acrílica termopolimerizável da marca Clássico® em muflas préconfeccionadas medindo $5 \times 5 \mathrm{~cm}$ de extensão e com altura de $1 \mathrm{~cm}$, seguindo as diretrizes do fabricante.

A polimerização desses corpos de prova seguiu o protocolo de polimerização da resina acrílica de ciclo longo onde leva-se a mufla ao banho-maria e eleva-se a temperatura da água a $70^{\circ} \mathrm{C}$ por 9 horas, seguida de $3 \mathrm{~h}$ a $100^{\circ}$ (Dos Santos, 2020).

Estes corpos de prova foram divididos em dois grupos: Sem biocida (G1) e com biocida (G2) em sua rede polimérica. Segundo o protocolo de Dos Santos, 2016, o grupo com biocida (G2) foi acrescido de $2 \mathrm{ml}$ de policloreto de dialildimetilamônio em sua massa.

Posteriormente, os corpos de prova foram cortados em formato redondo utilizando uma faca circular, com chanfro afiado à 75 graus e 30 milímetros de diâmetro interno. A ferramenta foi desenvolvida através do software Autodesk Inventor Professional 2016. A mesma foi acoplada em uma furadeira de bancada para o corte dos discos.

Com os corpos de provafinalizados, os testes de compressão foram realizados com auxílio da maquina de ensaio universal Instron3369 de coluna dupla, em movimento quasi-estático sendo capturadas imagens através da câmera Nikon D90, que possui 12.2 megapixels de resolução, captanto 80 framers/segundo, a partir do programa Control Pro, no modo time lapse shooting. O tempo de exposição foi ajustado para que as imagens ficassem mais claras, para isso, foi utilizado um sistema de iluminação LED, da Visual Instrumentation Corporation, operando com 50\% da capacidade máxima. O arranjo completo está representado na Figura 1.

Figura 1: Arranjo experimental para o teste de compressão.

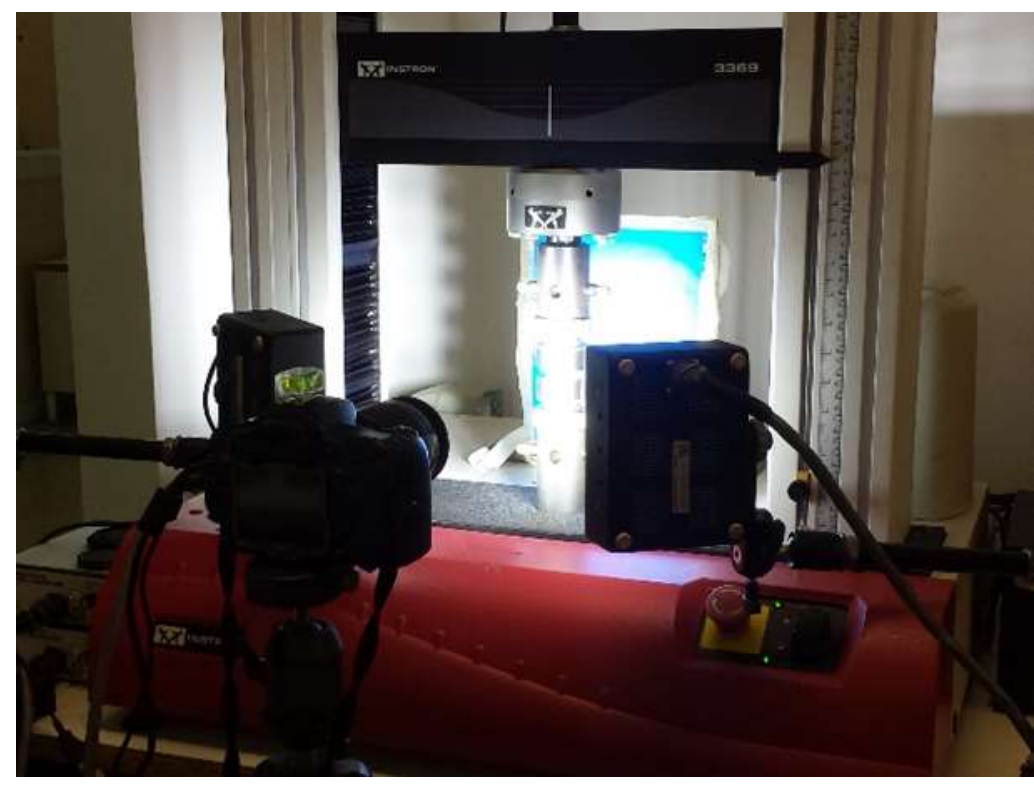

Fonte: Autores.

Para determinar a tensão verdadeira no corpo de prova, foi obtido o diâmetro instantâneo médio, podendo assim calcular a área da seção transversal média do corpo de prova, a qual está sujeita à tensão. O diâmetro instantâneo foi estimado a partir das fotografias tiradas pela câmera digital. Para isso, foi utilizado o programa MATLAB cuja função é analisar cada uma das fotografias e retornar o diâmetro médio para aquele instante específico. A análise se baseará na identificação de 
objetos que estão no primeiro plano, ou seja, à frente do plano de fundo. Isso é feito analisando a matiz de cores em cada pixel da figura e verificando quais pontos contrastam com a cor do plano de fundo.

As informações foram armazenadas seguindo o modelo RGB, modelo de cor aditivo, com fundo de cor azul onde as cores primárias são: vermelho (red), verde (green) e azul (blue), que quando combinados podem gerar todo o espectro de cores. A cor é representada como um vetor tridimensional (r, g, b), onde cada componente muda de zero para o valor máximo.

Após delimitar o espaço entre os pratos de compressão, o algoritmo localiza a área da imagem de ambos os lados e compara o valor da cor de fundo azul atribuída a cada pixel no modelo RGB com um limite inferior predeterminado, o qual está ligado ao valor médio do fundo de cor uniforme. O diâmetro aproximado do corpo é obtido pela diferença média (em pixels) entre os pontos das bordas esquerda e direita de uma mesma linha.

Por meio do software de controle da máquina, é possível visualizar e exportar os dados obtidos. A exportação é feita no formato Comma Separated Values (.csv), o que permite correlacionar a força de compressão (N) com o deslocamento vertical (mm). Os valores experimentais foram plotados no diagrama de dispersão com o auxílio do programa MATLAB. Os dados obtidos foram classificados com o auxílio do programa Excell 2007, e a análise estatística foi realizada utilizando o Biostatic 5.3 para traçar o diagrama de comportamento do material.

\section{Resultados}

Com os dados tabulados, foram evidenciados os resultados por meio de gráficos que mostram o comportamento dos materiais frente aos testes de compressão. Observando os gráficos 1 e 2 nota-se que G1 tem uma deformação mais lenta e exige mais força de compressão quando comparado com G2.

Gráfico 1: Teste no grupo G1, sem o biocida.

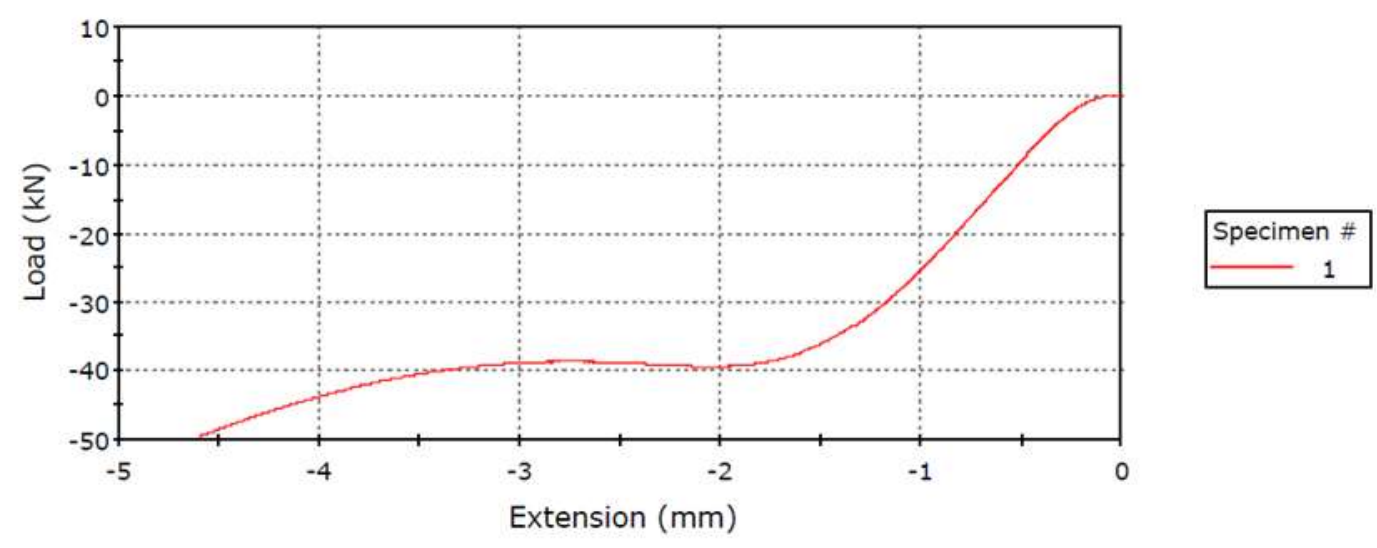

Fonte: Autores. 
Gráfico 2: Teste no grupo G2, com o biocida.

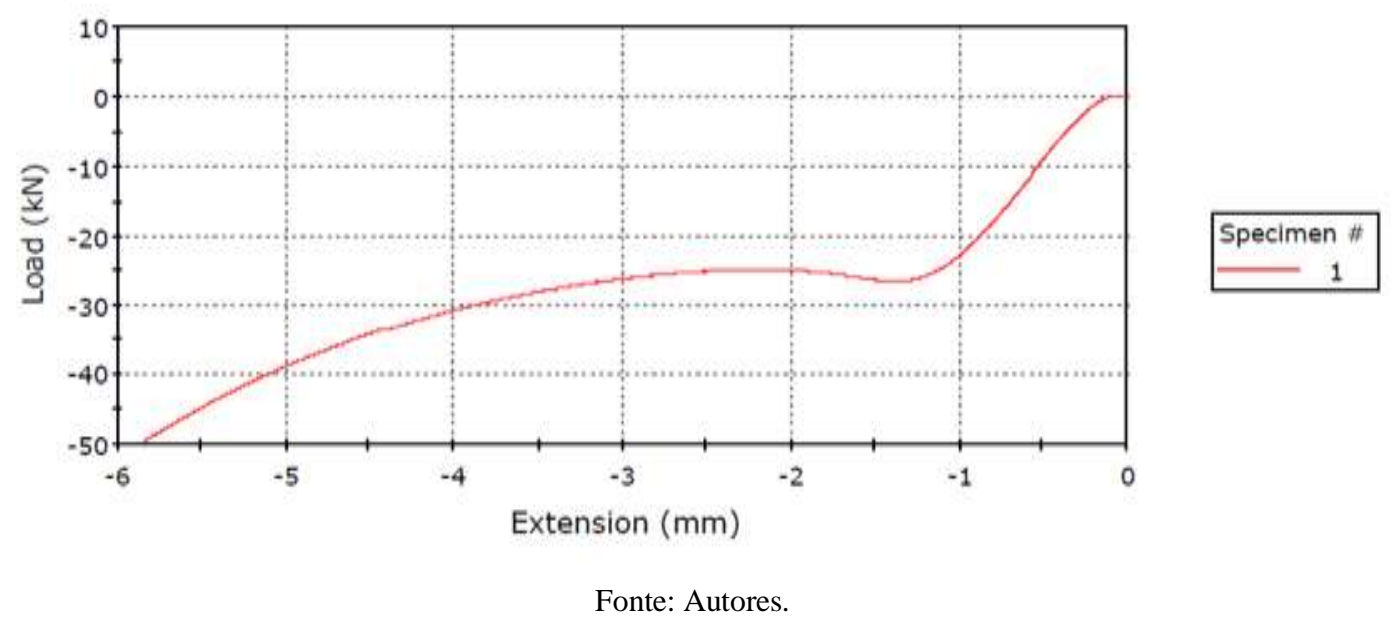

\section{Discussão}

A capacidade que polímeros têm de composição para melhorar suas características físicas, biológicas e mecânicas incita a realização de ensaios laboratoriais para esse fim. Esta pesquisa encontrou embasamento nas afirmações de Larsens em 2006, von Cramon-Taubadel N em 2011, Eyquem et al. em 2019, que afirmam que com a evolução humana sabe-se que a carga mastigatória sofreu uma redução em sua intensidade decorrente as mudanças dietéticas porém, mesmo com essa redução estudos devem ser realizados visando avaliar a possibilidade de utilização dos mais diversos materiais para confecção de próteses que resistam aos esforços mastigatórios, por exemplo.

Dos Santos et al. em 2019 e 2020 propôs o uso do poli(cloreto de dialildimetilamônio) agregado a resina acrílica termicamente ativada, material também utilizado na confecção de próteses bucomaxilofaciais. Os estudos realizados acerca da eficiência desse conjunto frente aos microrganismos obtiveram resultados positivo, mas o próprio autor recomenda apenas o uso desse material para próteses sem esforço mastigatório por não haver estudos conduzidos para esse fim, reforçando a necessidade do presente estudo

Os resultados desta pesquisa demonstraram que o biocida quando agregado a resina acrílica termicamente ativada,e observado em ambiente de tensão e deformação em teste mecânico de compressão apresenta uma deformação mais acentuada. Este fato se deu devido a adição de líquido na massa desse material que segundo Phillips em 1993, .Anusavice em 2000 e Camacho et al. em 2014 a relação correta entre seus componentes é de suma importância para a obtenção das propriedades ideais na estrutura final.Como este grupo de estudo já havia obtido sucesso, do ponto de vista biológico com a porcentagem utilizada, a intenção foi testar se a mesma proporção de poli(cloreto de dialildimetilamônio) incluído na resina traria uma melhora mecânica. Estudos com diferentes proporções entre os materiais e sua capacidade biológica e mecânica alinhadas, devem ser estimulados.

\section{Considerações Finais}

Após a inclusão do poli (cloreto de dialildimetilamônio) na resina acrílica termopolimerizadas ficou evidente que existem prejuízos nas propriedades mecânicas desse material. A partir das contribuições dessa pesquisa é necessária a realização de mais testes, referentes às propriedades físicas e mecânicas, para uma futura utilização ou não desse material em locais que exijam resistência a algum tipo de força sobre ele. 


\section{Agradecimentos}

Os autores agradecem pela bolsa de iniciação científica concedida pela fundação nacional de desenvolvimento do ensino superior particular (FUNADESP) para os discentes presentes nesse artigo.

\section{Referências}

Amal, N. A. \& Al, N. A. A. (2016) Antifungal Effect of Hennaa gainst Candida albicans Adhered to Acrylic Resin as a Possible Method for Prevention of Denture Stomatitis. International Journal of Environmental ResearchandPublic Health, 13(12), 520.

Anusavice, K. J. (2000) Phillips materiais dentários. (10a ed.), Guanabara Koogan.

Camacho, A. P., Svidzinski, T. I. E., Furlaneto, M. C. et al. (2014) Acrylic resins for dental use based polymethylmethacrylate. Brazilian Journal of Surgery and Clinical Research, 6(3), 63-72.

Coto, N. P., Geraldini, C. A. C., Dos Santos, R. L. O., Dias, R. B. E. (2017) Maxillofacial rehabilitation in a patient with conjunctival squamous cell carcinoma. Revista Brasileira De Cirurgia Da Cabeça E Pescoço (IMPRESSO), 46, 32-34,.

Da Silva, P. M. B. (2009) Efeito antimicrobiano das soluções desinfetantes sobre biofilme de C. Albicans em resinas acrílicas termopolimerizáveis. [Dissertação]. São Paulo: Faculdade de Odontologia, Universidade São Paulo.

Dos Santos, R. L. O., Sarra, G., Lincopan, N., Petri, D. F. S, Aliaga, J., Marques, M. M., Dias, R. B., Coto, N. P., Sugaya, N. N., Paula, C. R. (2020) Preparation, Antimicrobial Properties, and Cytotoxicity of Acrylic Resins Containing Poly(diallyldimethylammonium chloride). Int J Prosthodont, 26. 10.11607/ijp.6506.

Dos Santos, R. L. O. (2020) Estudo da eficiência antifúngica do biocida poli(cloreto dialildimetilamônico) incluído em resinas acrílicas a base de metilmetacrilato. [Tese]. São Paulo: Faculdade de Odontologia, Universidade de São Paulo.

Dos Santos, R. L. O,, Pedroso, V. B., Florenzi, G. P., Menezes, L. S. R., Sugaya, N. N., Paula, C. R. (2019) Antifungal efficiency of chemically and thermallyactivated acrylic resins after surface treatment using poly (diallyldimethylammonium chloride). Sociedade Brasileira De Medicina Tropical. Revista, 52 , 1-4.

Silva, M. J. A. (2012) Formação de biofilme de cândida albicans na superfície de uma resina acrílica termopolomerizavel tratada com polimento liquido. [Dissertação]. São Paulo: Faculdade de Odontologia, Universidade São Paulo.

Eisenburger, M., Riechers, J., Borchers, L. et al. (2008) Load-bearing capacity of direct four unit provisional composite bridges with fibre reinforcement. $J$ Oral Rehabil, 35,375-81.

Eyquem, A. P., Kuzminsky, S. C., Aguilera, J., Astudillo, W., \& Toro-Ibacache, V. (2019) Normal and altered masticatory load impact on the range of craniofacial shape variation: An analysis of pre-Hispanic and modern populations of the American Southern Cone. PLoS One, 14(12), e0225369. 10.1371/journal.pone.0225369.

Larsen, C.S. (2006) The agricultural revolution as environmental catastrophe: Implications for health and lifestyle in the Holocene. Quaternary International, $150(1), 12-20$.

Peracini, A. (2012) Soluções higienizadoras de prótese total: avaliação da remoção de biofilme e feito sobre propriedades da resina acrilica termopolimerizavel. [Tese]. Faculdade de Odontologia de Ribeirão Preto, Universidade de São Paulo.

Phillips, R. W. (1993) Skinner materiais dentários. (9a ed.), Guanabara Koogan, 334.

Rezende, J. R. V. (1997) Fundamentos da prótese buco-maxilo-facial. Sarvier.

Rocha, V., Dos Santos, R. L. O., Petri D. F. S., Dias, R. B., \& Coto, N. P. (2017) Uso de biocidas na superfície de matérias para confecção de próteses bucomaxilofaciais. Rev Cir Traumatol Buco-maxilo-fac. 17(1), 21-24.

Santos, R. L. O., França, A. M. S., Pereira, L., Dias, R. B., \& Orestes, S. (2016) Reabilitação com oftalmopróteses em dois pacientes com distintas etiologias de perda ocular. Revista de Cirurgia e Traumatologia Buco-Maxilo-Facial (Online), 16,57-61.

Straioto, F. G., Ricomini Filho, A. P., Fernandes Neto, A. J., \& Del Bel Cury, A. A. (2010) Polytetrafluorethylene added to acrylic resins: mechanical properties. Braz Dent J, 21,55-59

Turano, J. C., Turano, M. L., \& Turano, M. V. (2010) Fundamentos da prótese total. Editora Santos.

von Cramon-Taubadel, N. (2011) The relative efficacy of functional and developmental cranial modules for reconstructing global human population history. American Journal of Physical Anthropology, 146(1), 83-93. 